\title{
A Modified Nonstationary Tidal Harmonic Analysis Model for the Yangtze Estuarine Tides
}

\author{
Min GAN AND YONGPING CHEN \\ State Key Laboratory of Hydrology-Water Resources and Hydraulic Engineering, and College of Harbor, \\ Coastal and Offshore Engineering, Hohai University, Nanjing, China \\ SHUNQI PAN \\ School of Engineering, Cardiff University, Cardiff, United Kingdom \\ JIANGXIA LI AND ZIJUN ZHOU \\ State Key Laboratory of Hydrology-Water Resources and Hydraulic Engineering, and College of Harbor, \\ Coastal and Offshore Engineering, Hohai University, Nanjing, China
}

(Manuscript received 7 November 2018, in final form 18 January 2019)

\begin{abstract}
Influenced by river discharge, the tidal properties of estuarine tides can be more complex than those of oceanic tides, which makes the tidal prediction less accurate when using a classical tidal harmonic analysis approach, such as the T_TIDE model. Although the nonstationary tidal harmonic analysis model NS_TIDE can improve the accuracy for the analysis of tides in a river-dominated estuary, it becomes less satisfactory when applying the NS_TIDE model to a mesotidal estuary like the Yangtze estuary. Through the error source analysis, it is found that the main errors originate from the low frequency of tidal fluctuation. The NS_TIDE model is then modified by replacing the stage model with the frequency-expanded tidal-fluvial model so that more subtidal constituents, especially the "atmospheric tides," can be taken into account. The results show that the residuals from tidal harmonic analysis are significantly reduced by using the modified NS_TIDE model, with the yearly root-mean-square-error values being only $0.04-0.06 \mathrm{~m}$ for the Yangtze estuarine tides.
\end{abstract}

\section{Introduction}

Tides in oceanic and coastal waters are regarded as regular and predictable through the harmonic analysis of field tidal-level data (Matte et al. 2013). Based on the concept of equilibrium tides (Foreman 1977; Pawlowicz et al. 2002), it is assumed that the tidal levels can be represented by the sum of a series of cosine functions. Each of the cosine functions can be considered as one tidal constituent, having constant values of amplitude and phase. With no more than 150 tidal constituents being taken into account, the classical harmonic analysis model T_TIDE can predict the tidal levels by representing more than $90 \%$ signal variance of measured tides in the coastal zones (Pan et al. 2018a). However, when the tides propagate into an estuary, their interaction with the river discharge will make

\footnotetext{
Corresponding author: Yongping Chen, ypchen@hhu.edu.cn
}

the amplitudes and phases of tidal constituents nonstationary (Gallo and Vinzon 2005). This will significantly reduce the accuracy of T_TIDE model predictions in the river-influenced tidal regime.

The nonstationarity of tidal properties (amplitude and phase), due to the effect of river discharge, complicate the tidal harmonic analysis, which makes the tidal prediction rather difficult (Jay and Kukulka 2003; Kukulka and Jay 2003a,b; Matte et al. 2013, 2014; Guo et al. 2015; Hoitink and Jay 2016; Pan et al. 2018a,b). Guo et al. (2015) attempted to use a wavelet analysis approach to quantify the effect of river discharge on the tides in the Yangtze estuary. Their results showed that tidal signals can be altered differently by the river discharge in different seasons. Due to the restriction of Heisenberg uncertainty principle (Jay and Flinchem 1999; Flinchem and Jay 2000), the wavelet analysis approach can only be used to interpret the tidal signal variations within a given tidal band (i.e., the semidiurnal or diurnal tides) but not 
for individual tidal constituents. Alternatively, the time series of tidal-level data can be separated into a number of short-term periods, within which the tides are assumed as quasi stationary, so that the T_TIDE model can be applied. This is known as the short-term harmonic analysis approach as used by Jay and Flinchem (1999) and Guo et al. (2015). Due to the segmentation of field data and the restriction of Rayleigh criterion (Jay and Flinchem 1999), a trade-off between the resolution in time domain and the number of tidal constituents to be resolved in the short-term periods is required. If the nonstationarity of river discharge is very strong, the short-term harmonic analysis approach may generate inaccurate results due to the nonlinear effect between nearby tidal spectral bands (Guo et al. 2015). Considering the complexity of tidal-fluvial regimes, the abovementioned approaches may not be able to reveal all the information for the estuarine tides which are strongly affected by the river discharge. Therefore, as suggested by Hoitink and Jay (2016), it is necessary to combine the commonly used analysis tools for stationary components and specialized tools for nonstationary components to accurately analyze the tides in estuaries.

Based on the previous studies by Jay (1991), Kukulka and Jay (2003a,b), and Jay et al. (2011), Matte et al. (2013) developed a nonstationary tidal harmonic analysis model, named NS_TIDE, to analyze the tides in river-dominated estuaries. The accuracy of the model is well validated for the Columbia River (Matte et al. 2013; Pan et al. 2018a,b), St. Lawrence fluvial estuary (Matte et al. 2014, 2018), and Pearl estuary (Cai et al. 2018; Zhang et al. 2018). The main improvement of the NS_TIDE model over the T_TIDE model was demonstrated in the analysis of estuarine tides in the upper tidal reach where the river discharge significantly influenced the tides. However, one of the source frameworks in the NS_TIDE model is based on the results of Kukulka and Jay (2003b), assuming that the tidal discharge is relatively smaller than the river discharge. This may limit its application in the tidal reach where both the river discharge and tidal discharge are strong and have similar magnitude.

Besides the harmonic analysis approach, the artificial neural network (ANN) approach has also been commonly used for the tidal analysis in coasts and estuaries (Tsai and Lee, 1999; Chang and Chen 2003; Chang and Lin 2006; Liang et al. 2008; Khan et al. 2016). This approach does not require any specific assumptions to be made for the mathematical form of tidal levels, but it adopts a learning process based on the training of historical dataset, enabling it to simulate a nonlinear complex system (Tsai and Lee 1999;
Khan et al. 2016). It can achieve the same or even higher accuracy than the harmonic analysis approach if the data for the learning process are sufficient and accurate enough. However, the data sufficiency is not easily satisfied for those estuaries that have not been intensively studied, and the data quality for the ANN learning process can also be easily influenced by the natural environmental changes as well as human activities in the study area (ASCE 2000a). These factors could limit the general use of the ANN approach in the tidal analysis. Therefore, the ANN approach can be considered as a good supplement (ASCE 2000b), but it may not be able to overtake the traditional harmonic analysis approaches for the tidal analysis.

The main objective of this study is to test the accuracy of the nonstationary tidal harmonic analysis model (NS_TIDE) in predicting the tides in the Yangtze estuary. The performance of the NS_TIDE model is carefully investigated at several hydrographic stations in the Yangtze estuary. Based on the error source analysis, both the stage model and the tidal-fluvial model in the NS_TIDE model are modified so that more subtidal constituents can be taken into account. The accuracy of the modified NS_TIDE model, herein named MNS_TIDE, is well examined by hourly continuous field data of 4 years at those hydrographic stations.

\section{Model description}

The main difference between the T_TIDE, NS_TIDE, and MNS_TIDE models is in the description of tidal levels for the harmonic analysis. The details are described as follows.

\section{a. T_TIDE model}

In the classical harmonic analysis model, the tidallevel $\eta(t)$ (Pan et al. 2018b) is described as

$$
\eta(t)=S+\sum_{k=1}^{n} H_{k} \cos \left(\sigma_{k} t-\varphi_{k}\right)
$$

where $t$ is the time; $S$ is the mean tidal level; $k$ is the index of tidal constituents; $H_{k}, \sigma_{k}$, and $\varphi_{k}$ are the amplitude, frequency, and phase of the $k$ th tidal constituent, respectively; and $n$ is the number of tidal constituents to be considered.

Equation (1) can be also written as the following:

$$
\eta(t)=S+\sum_{k=1}^{n}\left[c_{k} \cos \left(\sigma_{k} t\right)+s_{k} \sin \left(\sigma_{k} t\right)\right]
$$




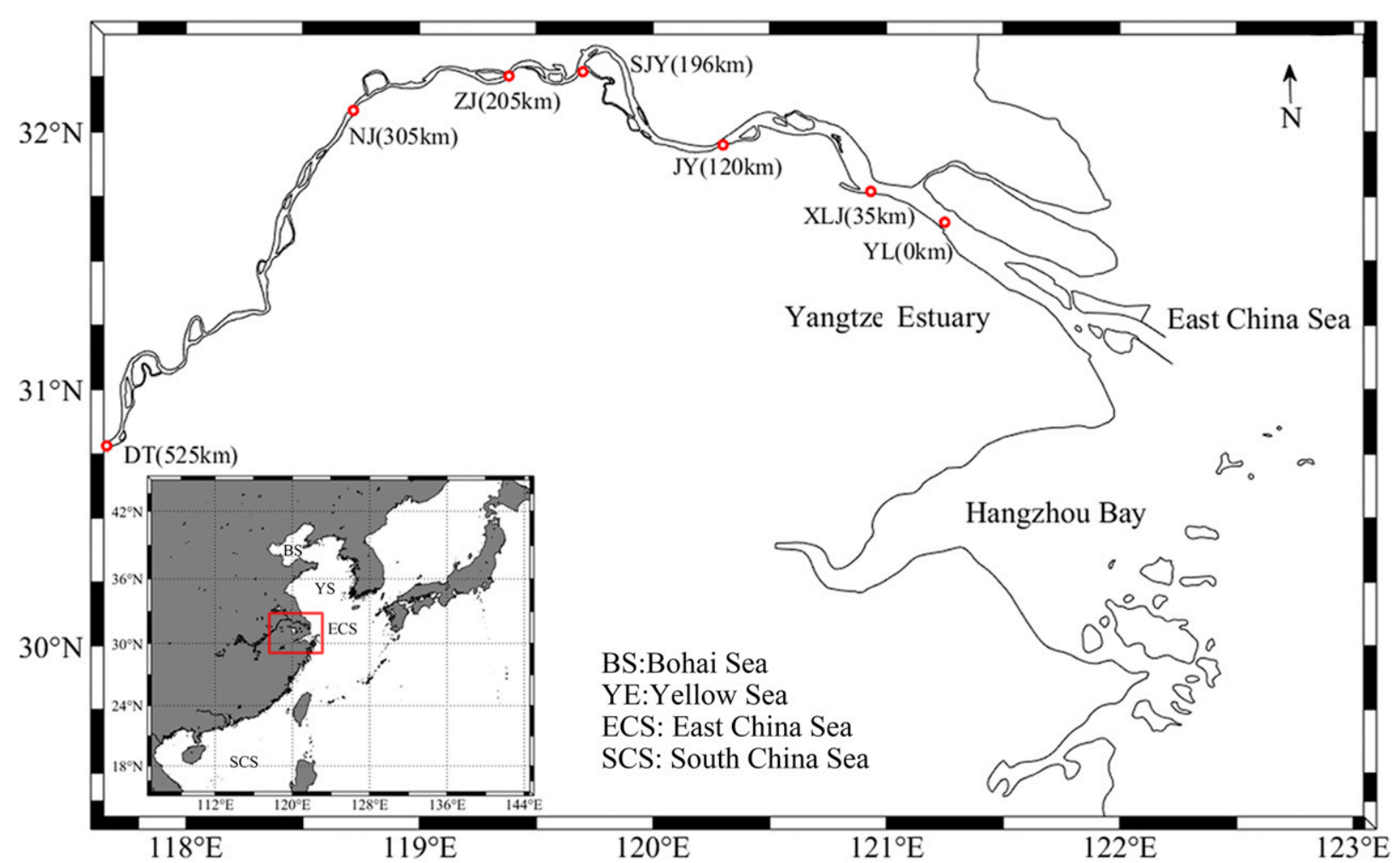

FIG. 1. Schematic diagram of the Yangtze estuary and the location of the hydrometric stations.

where $c_{k}$ and $s_{k}$ are the parameters related to the amplitude and phase of the $k$ th tidal constituent. The following relationships hold:

$$
\begin{aligned}
& H_{k}=\sqrt{c_{k}^{2}+s_{k}^{2}}, \\
& \varphi_{k}=\arctan \left(\frac{s_{k}}{c_{k}}\right),
\end{aligned}
$$

where $S, c_{k}$, and $s_{k}$ can be determined by the least squares method (Pawlowicz et al. 2002).

\section{b. NS_TIDE model}

When the tides propagate into the estuaries, the effect of the river discharge should be taken into account. In the NS_TIDE model, the tidal-level $\eta(t)$ is described as (Matte et al. 2013)

$$
\eta(t)=\underbrace{S\left[Q_{R}(t), R(t)\right]}_{\text {Stage model }}+\underbrace{\sum_{k=1}^{n}\left[c_{k}\left[Q_{R}(t), R(t)\right] \cos \left(\sigma_{k} t\right)+s_{k}\left[Q_{R}(t), R(t)\right] \sin \left(\sigma_{k} t\right)\right]}_{\text {Tidal-fluvial model }},
$$

where

$$
S\left[Q_{R}(t), R(t)\right]=S_{0}+S_{1} Q_{R}^{p_{s}}\left(t-t_{Q}\right)+S_{2} \frac{R^{q_{s}}\left(t-t_{R}\right)}{Q_{R}^{r_{s}}\left(t-t_{Q}\right)},
$$

(6) in which $\left(p_{s}, q_{s}, r_{s}\right)$ and $\left(p_{f}, q_{f}, r_{f}\right)$ are two sets of un$c_{k}\left[Q_{R}(t), R(t)\right]=c_{k, 0}+c_{k, 1} Q_{R}^{p_{f}}\left(t-t_{Q}\right)+c_{k, 2} \frac{R^{q_{f}}\left(t-t_{R}\right)}{Q_{R}^{r_{f}}\left(t-t_{Q}\right)}$,

$$
s_{k}\left[Q_{R}(t), R(t)\right]=s_{k, 0}+s_{k, 1} Q_{R}^{p_{f}}\left(t-t_{Q}\right)+s_{k, 2} \frac{R^{q_{f}}\left(t-t_{R}\right)}{Q_{R}^{r_{f}}\left(t-t_{Q}\right)},
$$
known exponents in the stage model and tidal-fluvial model, respectively; $Q_{R}(t)$ is the time series of lowpassed upstream river discharge with the frequency less than 0.03 cycle per hour ( $\mathrm{cph}) ; R(t)$ is the time series of greater diurnal tidal range at the reference station 


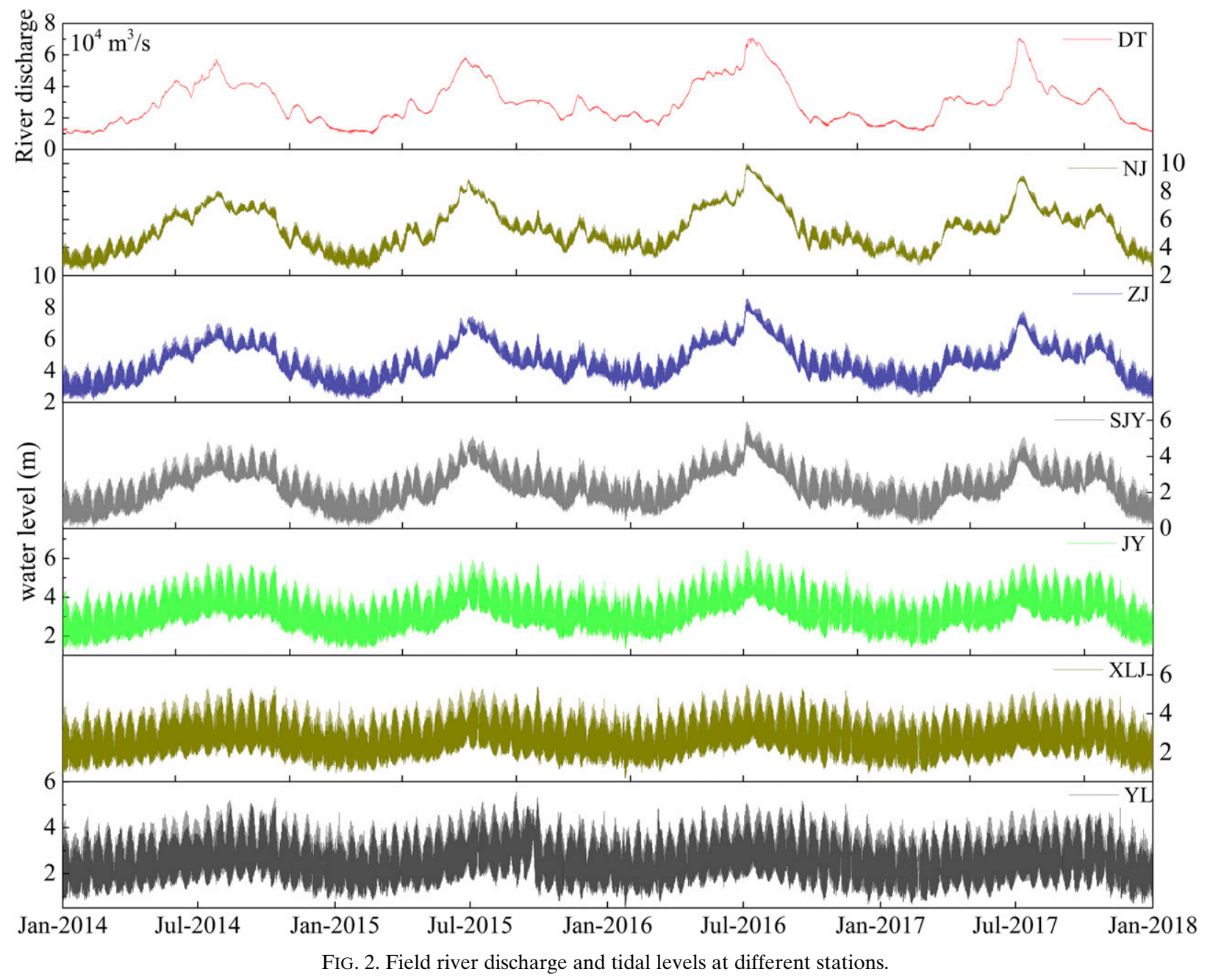

where tides are only slightly influenced by or under no influence of the river discharge; $t_{Q}$ is the time lag of the average time of river discharge propagating to the analysis station; and $t_{R}$ is the time lag of the average time of tidal waves at the reference station propagating to the analysis station. Here, $S_{i}, c_{k, i}$, and $s_{k, i}(i=0,1,2)$ are unknown parameters.

In the NS_TIDE model, the time lag $t_{Q}$ can be derived by seeking the maximum correlation between $Q_{R}(t)$ and the low-pass-filtered field tidal levels, while the time lag $t_{R}$ can be derived by seeking the maximum correlation between $R(t)$ and the range-filtered field tidal levels. The exponents $p_{s}, q_{s}$, and $r_{s}$ in the stage model can be solved by an iterative procedure using the time series of field tidal levels. The exponents $p_{f}$, $q_{f}$, and $r_{f}$ in the tidal-fluvial model can be solved by the same iterative procedure but using the highpassed (frequency is in the range of $0.03-0.05 \mathrm{cph}$ ) time series of field tidal levels. Once these exponents are determined, $S_{i}, c_{k, i}$, and $s_{k, i}(i=0,1,2)$ can be solved by the iteratively reweighted least squares analysis method (Holland and Welsch,1977; Codiga 2011; Leffler and Jay 2009). More details about the NS_TIDE model can be found in Matte et al. (2013, 2014).

\section{c. MNS_TIDE model}

As described above, the tidal-fluvial model of NS_TIDE can be used to account for the common tidal fluctuation (frequency is between 0.03 and $0.05 \mathrm{cph}$ ), while the stage model of NS_TIDE is used to account for the subtidal fluctuation (frequency is less than $0.03 \mathrm{cph}$ ) of tidal levels. Due to the complexity in analytical solutions of the stage model, the theoretical framework of the stage model, derived by Kukulka and Jay (2003b), assumes that the tidal discharge be considerably smaller than the river discharge so that the theoretical framework of the stage model can be simplified to some extent. However, this may deteriorate the accuracy of the stage model in the analysis of the tidal 


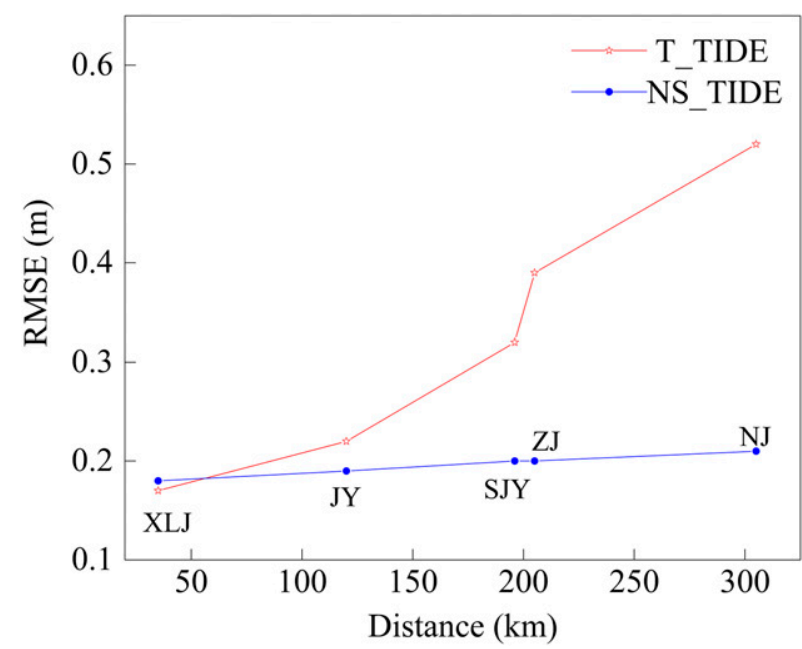

FIG. 3. Comparison of the RMSE values of the predicted results of T_TIDE and NS_TIDE toward the upstream direction.

signal in a mesotidal estuary like the Yangtze estuary. The results of Pan et al. (2018a) show that the main errors of NS_TIDE are from the stage model, which further indicates that NS_TIDE cannot well capture the subtidal fluctuation of estuarine tides. In fact, the previous studies show that the amplitudes of subtidal constituents in the upper part of the Yangtze estuary are quite large (Guo et al. 2015; Tan et al. 2016); therefore, this study proposes a modified model MNS_TIDE from the NS_TIDE model for a better accounting for the subtidal fluctuation of tidal levels in the Yangtze estuary.

In the MNS_TIDE model, the tidal-level $\eta(t)$ is described as

$$
\begin{aligned}
\eta(t)= & S+\sum_{k=1}^{n}\left\{c_{k}\left[Q_{R}(t), R(t)\right] \cos \left(\sigma_{k} t\right)\right. \\
& \left.+s_{k}\left[Q_{R}(t), R(t)\right] \sin \left(\sigma_{k} t\right)\right\},
\end{aligned}
$$

in which $S$ is the mean tidal level, and the meanings of other parameters are the same as those in Eq. (5). In comparison with the NS_TIDE model, three main changes have been made. First, the stage model is simplified, while the limitation of the frequency band in the tidal-fluvial model is expanded from $0.03-0.05$ to $0-0.5 \mathrm{cph}$. Second, more subtidal constituents, especially the "atmospheric tides," are included in the tidal-fluvial model. In total, 306 tidal constituents suggested by Tan et al. (2016) are used in the MNS_TIDE model. Third, to save the computational costs, the theoretical solutions of $p_{f}, q_{f}$, and $r_{f}$ derived by Kukulka and Jay (2003a) are directly used in Eqs. (7) and (8). They are equal to 1, 2, and $1 / 2$, respectively. Preliminary sensitivity tests (although the results are not shown here) prove that the accuracy is not much affected by this change compared to the one
TABLE 1. Estimated time lags and the numbers of selected tidal constituents in NS_TIDE.

\begin{tabular}{lcccccc}
\hline \hline Stations & $t_{Q}(\mathrm{~h})$ & $t_{R}(\mathrm{~h})$ & 2014 & 2015 & 2016 & 2017 \\
\hline XLJ & 3 & 6 & 39 & 32 & 30 & 31 \\
JY & 16 & 3 & 39 & 32 & 30 & 29 \\
SJY & 18 & 4 & 39 & 31 & 28 & 32 \\
ZJ & 3 & 5 & 37 & 32 & 28 & 32 \\
NJ & 3 & 6 & 38 & 29 & 29 & 26 \\
\hline
\end{tabular}

obtained by the iterative procedure as suggested by Matte et al. (2013).

\section{Research domain and observation data}

The Yangtze estuary is the regime where the Yangtze River meets the East China Sea (Fig. 1). It is characterized by large river discharge, strong tides, and moderate winds and waves (Guo et al. 2015). The variations of river discharge are significant, with recorded maximum and minimum river discharges of 84000 and $6730 \mathrm{~m}^{3} \mathrm{~s}^{-1}$, respectively (Lu et al. 2015). Semidiurnal tides are dominant at the river mouth of the Yangtze estuary, with the mean tidal range of $2.65 \mathrm{~m}$ (Chen et al. 2016).

The field data measured at several hydrographic stations (Fig. 1) along the Yangtze estuary are collected for this study. The Datong (DT) station is the most upstream station, where the river discharge is almost not affected by the tides, so the river discharge data at the DT station can be used as the upstream river discharge for the Yangtze estuary. The Yanglin (YL) station is the most seaward station, where the tidal levels are only marginally influenced by the river discharge, so the YL station is considered as the reference station for the nonstationary tidal harmonic analysis. The tidal-level data measured at the YL station are used to determine the tidal ranges downstream. The location of the YL station is also taken as the origin point to calculate the distances of other hydrographic stations, while the upstream direction is specified as the positive direction. The hourly tidal levels measured at six stations [i.e., YL $(0 \mathrm{~km})$, Xuliujing (XLJ; $\sim 35 \mathrm{~km}$ ), Jiangyin (JY; $\sim 120 \mathrm{~km}$ ), Sanjiangying (SJY; $\sim 196 \mathrm{~km}$ ), Zhenjiang (ZJ; $\sim 205 \mathrm{~km})$, and Nanjing (NJ; $\sim 305 \mathrm{~km})$ ] are synchronized with the hourly river discharge data at the DT $(\sim 525 \mathrm{~km})$ station during the period of 1 January 2014 to 31 December 2017. As shown in Fig. 2, the influence of the river discharge on tides becomes significant in the upper part of the Yangtze estuary, including the JY, SJY, ZJ, and NJ stations. All the observation data will be used to examine the performances of the T_TIDE, NS_TIDE, and MNS_TIDE models, in terms of the root-mean-square error (RMSE) 


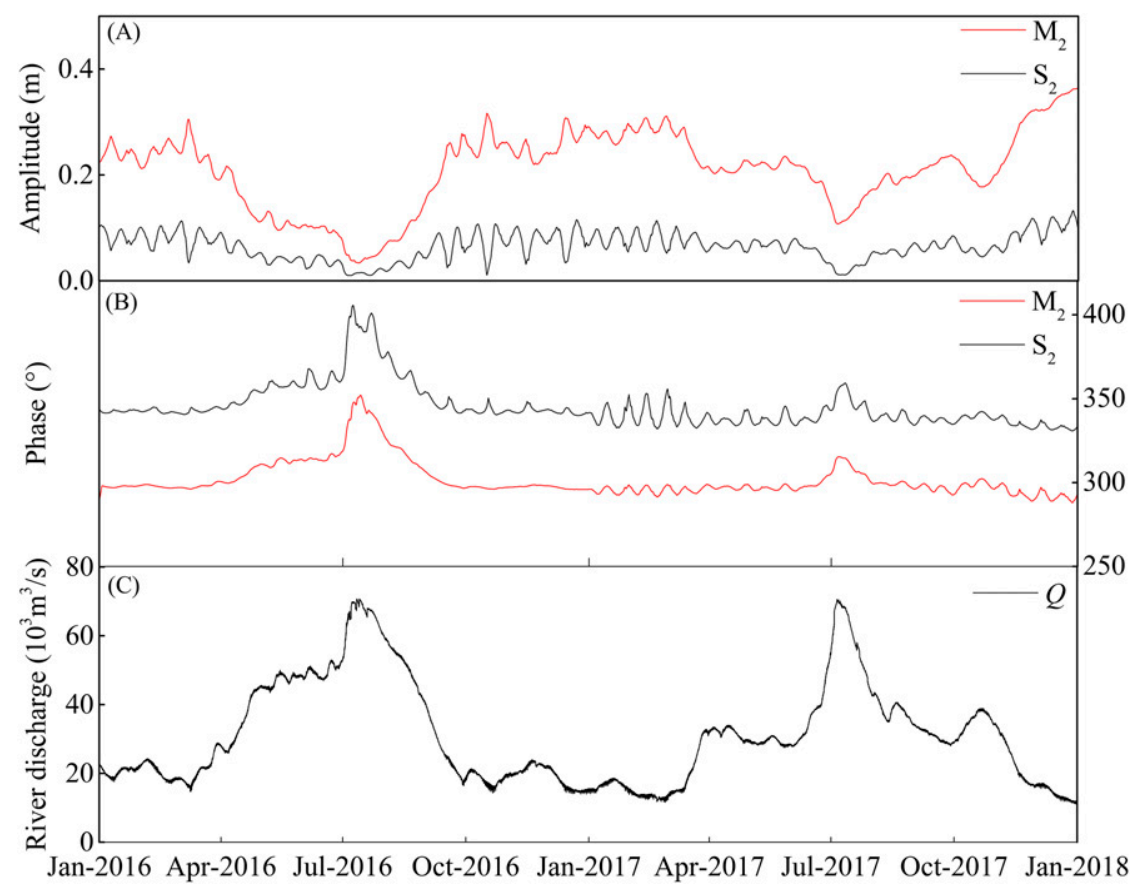

FIG. 4. The variation of amplitudes and phases of $\mathrm{M}_{2}$ and $\mathrm{S}_{2}$ tidal constituents with upstream river discharge.

of predicted tidal levels, at different locations of the Yangtze estuary.

\section{Results}

a. The performance of $T \_T I D E$ and NS_TIDE

Due to the nature of T_TIDE, the time series used in the tide analysis should be sufficiently long so that enough tidal constituents can be considered for the analysis but, on the other hand, not too long to ensure the stationary tidal properties to be maintained. Following the suggestions by Jay and Flinchem (1999) and Guo et al. (2015), the short-term tidal harmonic analysis approach was adopted in the T_TIDE model. The measured data were divided into yearly time series (exactly 8785 points). For comparison, the

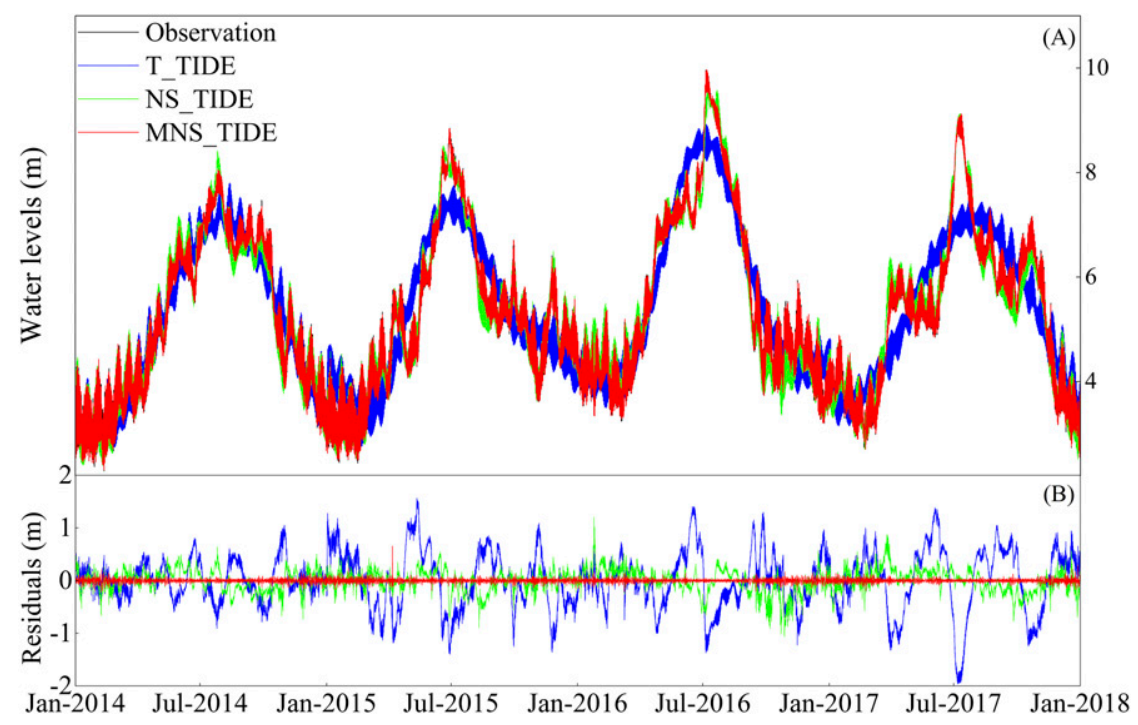

FIG. 5. (a) The comparisons of the field tidal levels in NJ station and the predicted results calculated by T_TIDE, NS_TIDE, and MNS_TIDE and (b) the related residuals. 


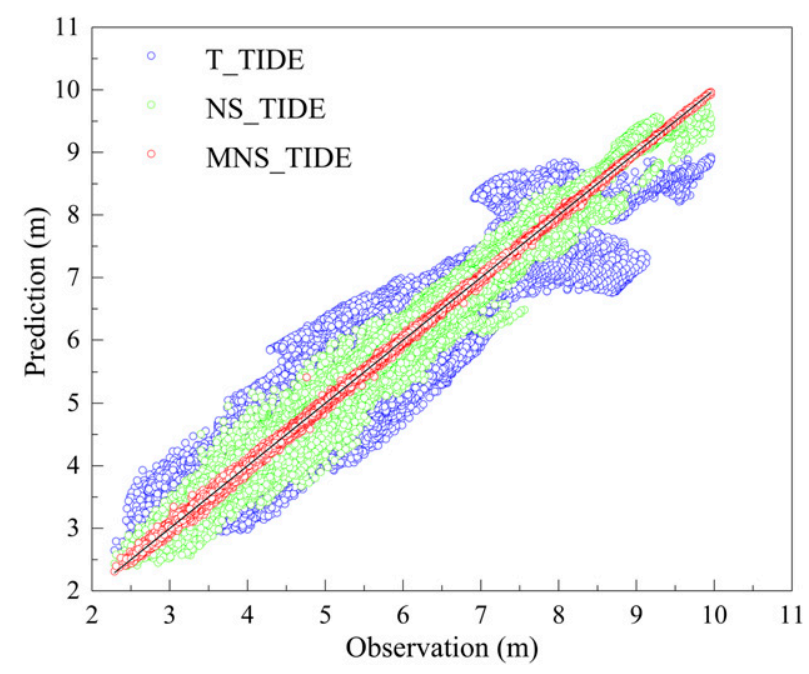

FIG. 6. The comparison of all the field tidal levels with the predicted results calculated by T_TIDE, NS_TIDE, and MNS_TIDE.

same approach was also adopted in the NS_TIDE model.

As described above, the YL station is chosen as the reference station in the NS_TIDE model. Preliminary results of tidal harmonic analysis show that the tidal properties at the YL station are quite stationary, with only little influence of the river discharge. The parameter $\eta$ for the redefined Rayleigh criterion (Matte et al. 2013) in the NS_TIDE model is set as 0.15. The significance of tidal constituents is determined by a correlated noise model, with the mean signal-to-noise ratio no less than 2. Other parameters' settings are the same as Matte et al. (2013) specified in the NS_TIDE model. The RMSE values of the predicted tidal levels of the T_TIDE and NS_TIDE models were calculated to illustrate the model accuracy versus the field tidal levels.

Figure 3 shows the comparisons of the RMSE results between the T_TIDE and NS_TIDE models. At the XLJ station, the RMSE value of the T_TIDE model is $0.17 \mathrm{~m}$ and the RMSE value of the NS_TIDE model is $0.18 \mathrm{~m}$, which shows the NS_TIDE model has the same magnitude of accuracy as the T_TIDE model in the lower reach of the Yangtze estuary; however, when the influence of the river discharge becomes more significant, the advantage of NS_TIDE over T_TIDE appears. A significant increase of RMSE is shown toward the upstream direction in the T_TIDE model, with the value of $0.52 \mathrm{~m}$ at the $\mathrm{NJ}$ station, while only a moderate increase of RMSE is shown in the NS_TIDE model, with the value of $0.21 \mathrm{~m}$ at the $\mathrm{NJ}$ station.

To more accurately estimate time lags $t_{Q}$ and $t_{R}$, the whole time series rather than the yearly time series was
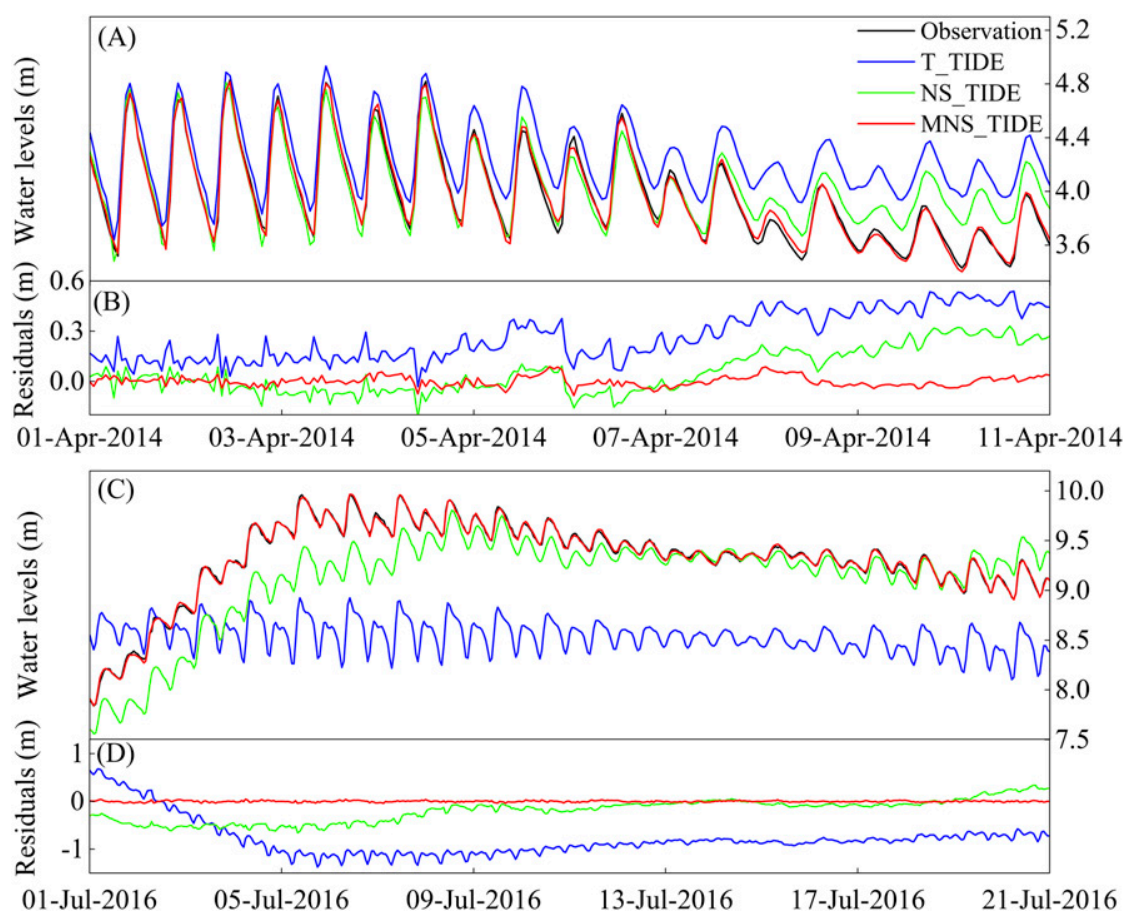

FIG. 7. The comparison of the field tidal levels with the predicted results calculated by T_TIDE, NS_TIDE, and MNS_TIDE in (a),(b) the dry season of 2014 and (c),(d) the flood season of 2016. 


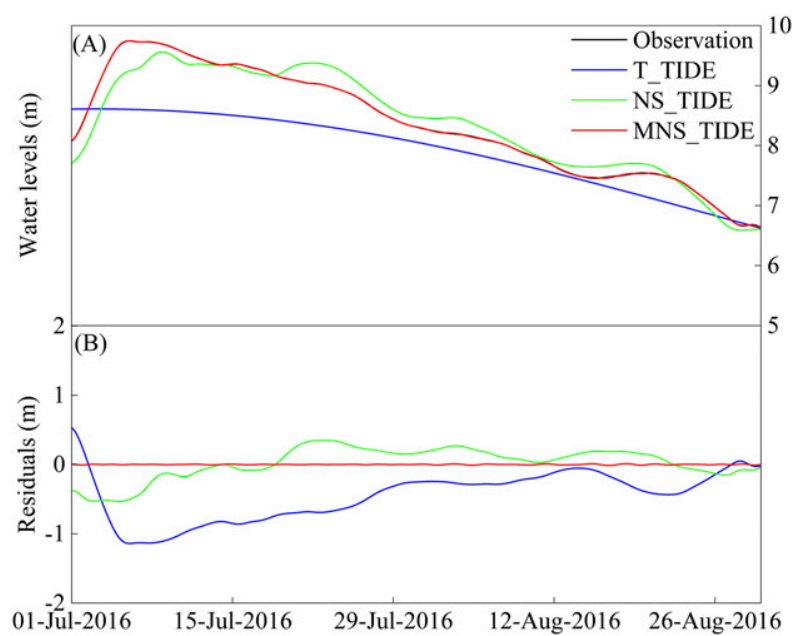

FIG. 8. (a) The comparison of the low-passed field tidal levels with the low-passed predicted results calculated by T_TIDE, NS_TIDE, and MNS_TIDE and (b) the related residuals in the flood season of 2016.

used to calculate the maximum correlation. The calculated time lags $t_{Q}$ and $t_{R}$ and the number of selected tidal constituents at the individual stations in the Yangtze estuary are shown in Table 1 . Theoretically, toward the upstream direction, the time lag $t_{Q}$ should follow a decreasing trend, while $t_{R}$ should follow an increasing trend. However, surprisingly, the trend of the results is fairly random. It is noticed from Table 1 that the number of tidal constituents selected in the NS_TIDE model in 2014 and 2015 are more than the number in 2016 and 2017. This is because the seasonal variations of the river discharge in 2016 and 2017 are different from those in 2014 and 2015. It can be observed from Fig. 2 that the peak river discharges in the flood seasons of 2016 and 2017 are much larger than those in the flood seasons of 2014 and 2015. Different river discharge conditions makes tidal constituents under different effects of frictional dissipation (Kukulka and Jay 2003a). Therefore, the number of tidal constituents selected in the NS_TIDE model varies in different years.

The results at the NJ station are used for the further illustration of the nonstationary impact of the river discharge on the tidal properties in the Yangtze estuary. Figure 4 presents the amplitude and phase variations of two main tidal constituents, that is, $\mathrm{M}_{2}$ and $\mathrm{S}_{2}$, in different seasons. In the dry season (from November to April), the largest amplitudes of $\mathrm{M}_{2}$ and $\mathrm{S}_{2}$ tidal constituents reach 0.36 and $0.13 \mathrm{~m}$, while they reduce to 0.09 and $0.01 \mathrm{~m}$ in the flood season (from May to October), which indicates the river discharge in the flood season can significantly damp the energy of tidal constituents. Figure 4 shows that the river discharge can also affect the phases of $\mathrm{M}_{2}$ and $\mathrm{S}_{2}$ tidal constituents. The large river discharge can delay the propagation speed of tidal waves toward the upstream direction. It confirms the reasonability of NS_TIDE, which allows the amplitudes and phases of tidal

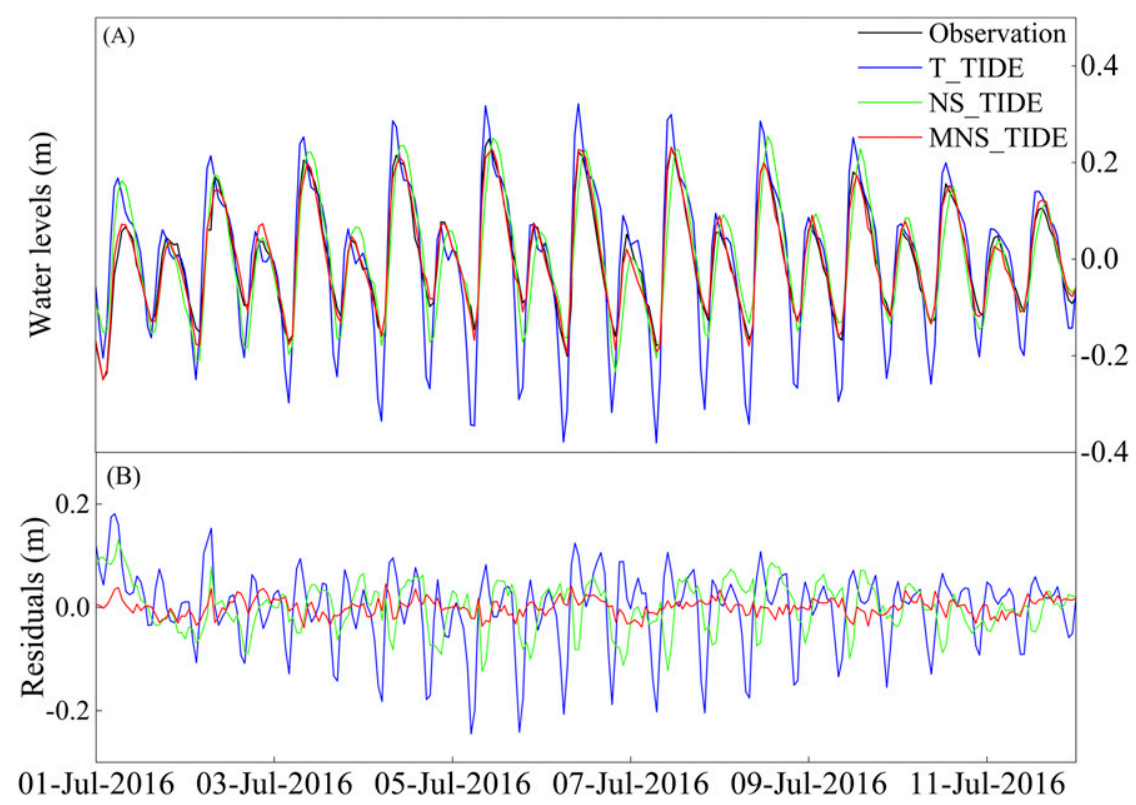

FIG. 9. (a) The comparison of the high-passed field tidal levels with the high-passed predicted results calculated by T_TIDE, NS_TIDE, and MNS_TIDE and (b) the related residuals in the flood season of 2016 . 

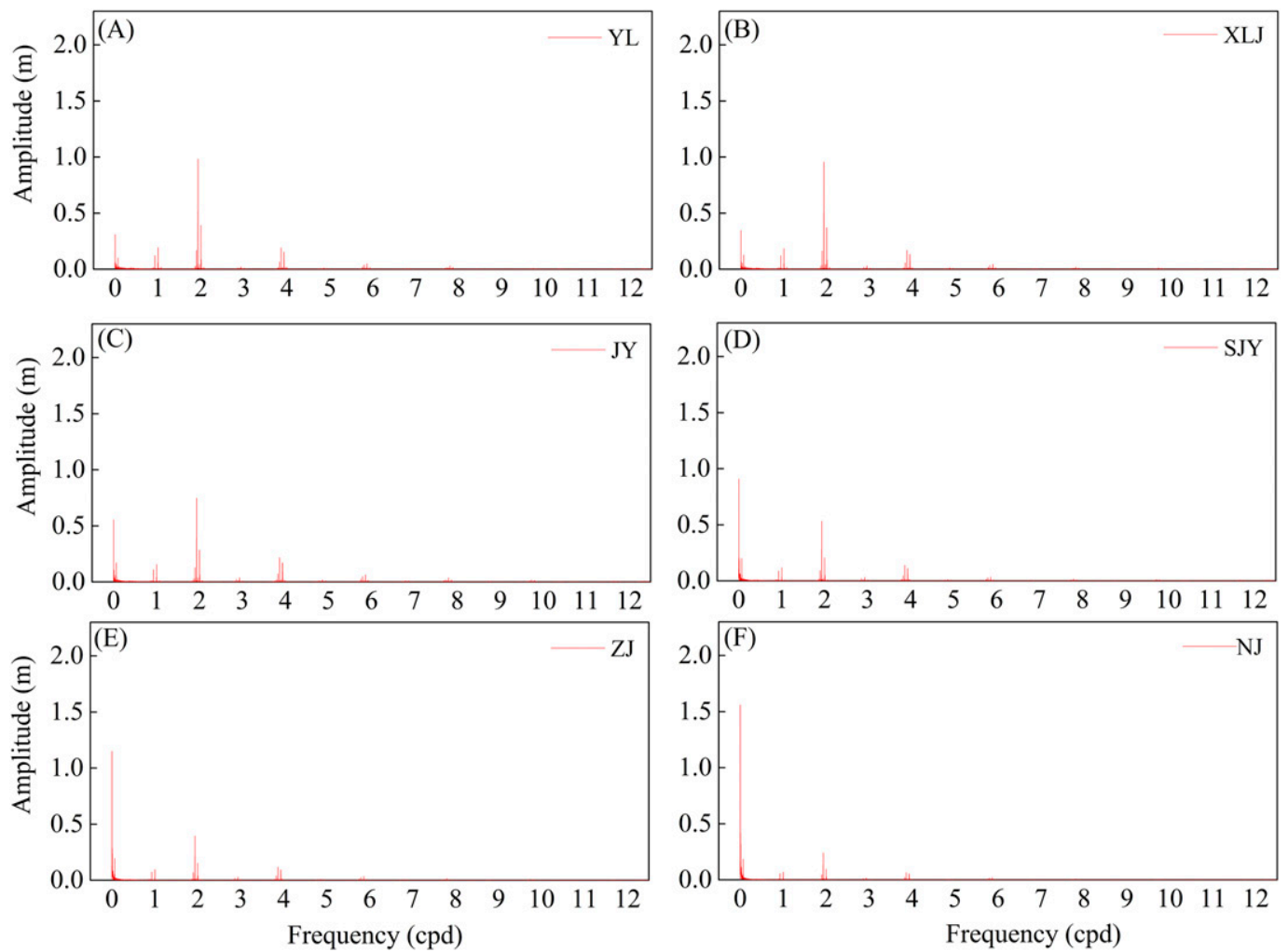

FIG. 10. The spectral amplitude distribution of the field tidal levels at (a) YL, (b) XLJ, (c) JY, (d) SJY, (e) ZJ, and (f) NJ stations calculated by Fourier analysis.

constituents to be nonstationary, following with the change of the river discharge upstream.

\section{b. The performance of MNS_TIDE}

The YL station is selected as the reference station, and the analysis data are divided into yearly time series (exactly 8785 points) in the MNS_TIDE model, same as in the T_TIDE and NS_TIDE models.

The comparison of the predicted and field tidal levels and their residuals at the $\mathrm{NJ}$ station is shown in time series in Fig. 5 and in a scatter diagram in Fig. 6. Both figures clearly show that the accuracy of MNS_TIDE is the best among the three models, with the yearly RMSE values being only $0.04-0.06 \mathrm{~m}$. To illustrate in detail, Fig. 7 presents the comparison of the predicted and field tidal levels and their residuals at the NJ station in the dry season of 2014 and the flood season of 2016. In comparison with the residuals shown in Figs. $7 \mathrm{~b}$ and $7 \mathrm{~d}$, it can be observed that the errors of the NS_TIDE model in flood seasons are larger than those in dry seasons, while the MNS_TIDE model makes a significant improvement over the NS_TIDE model in both dry and flood seasons.

To examine the performance of MNS_TIDE in representing the subtidal fluctuation of tidal levels in the Yangtze estuary, the predicted and field tidal levels were low passed and high passed to separate the low-frequency (subtidal band, below $0.03 \mathrm{cph}$ ) fluctuation and high-frequency (higher than subtidal frequency, above $0.03 \mathrm{cph}$ ) fluctuation of the tidal signals. Figures 8 and 9 present the comparisons of the lowpassed and the high-passed time series of tidal levels and their residuals at the NJ station in 2016, respectively. In comparison with the residuals, the main errors of the NS_TIDE model originate from the low-frequency (subtidal band) fluctuation, while the MNS_TIDE model can perfectly represent the subtidal fluctuation; thus, the errors of the MNS_TIDE model are significantly reduced.

\section{c. Fourier analysis of field data and residuals}

To further analyze the error sources of the NS_TIDE and MNS_TIDE models, the Fourier analysis of field data and residuals of each model was carried out. Figure 10 presents the distribution of amplitude spectrum of the field tidal levels in frequency domain. It can be seen that at the YL, XLJ, and JY stations, the frequency band around 2 cycles per day (cpd) is dominant. This is because they are in the lower reach of the Yangtze estuary, where the semidiurnal tides are dominant (Guo et al. 2015). However, toward the upstream 

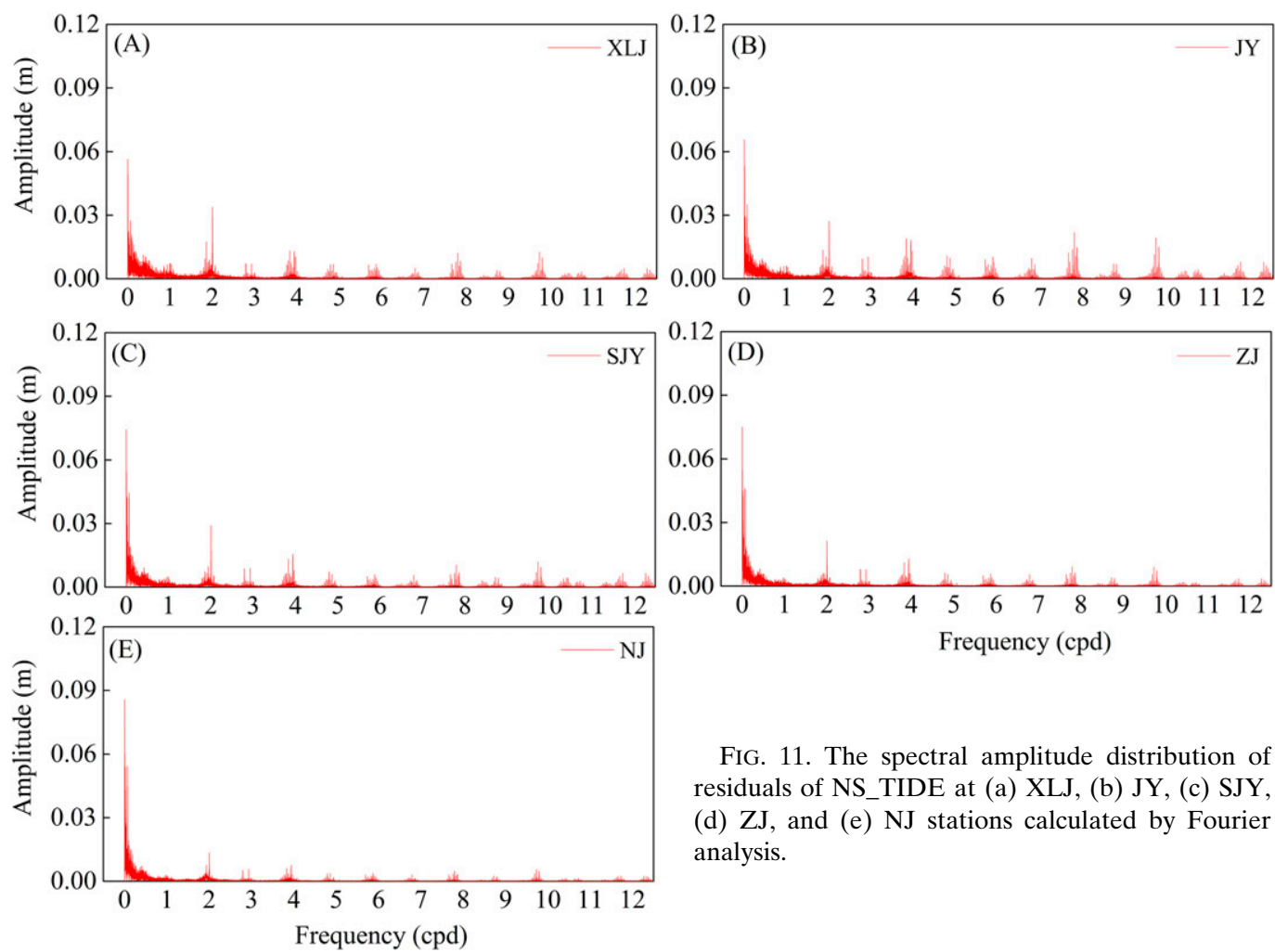

FIG. 11. The spectral amplitude distribution of residuals of NS_TIDE at (a) XLJ, (b) JY, (c) SJY, (d) ZJ, and (e) NJ stations calculated by Fourier analysis.

direction, the frequency band near $0.0027 \mathrm{cpd}$ (frequency of Sa tidal constituent) has a significant increase and becomes dominant at the SJY, ZJ, and NJ stations.

Figure 11 presents the distribution of residual amplitude spectrum of the NS_TIDE model in the frequency domain. It can be seen that the residual spectral amplitudes at the frequencies of $0-0.03 \mathrm{cph}$ are more significant than those at other frequencies, with the peak occurring at the frequency of $0.0027 \mathrm{cpd}$. This confirms that the errors of the NS_TIDE model mainly come from the subtidal band, particularly near the frequency of the Sa tidal constituent. In contrast, Fig. 12 presents the distribution of residual amplitude spectrum of the MNS_TIDE model in the frequency domain. The amplitude spectrum of the MNS_TIDE model is much smaller and more evenly distributed than that of the NS_TIDE model. This shows that the MNS_TIDE model is superior to the NS_TIDE model in the analysis of tidal constituents with widely distributed frequencies.

\section{Discussion}

As the river discharge has low-frequency periods such as annual and seasonal periods, its influence on tidal levels is mainly in the low-frequency range. The influence of the river discharge on tidal levels becomes stronger toward the upstream direction. Therefore, the spectral amplitude distribution of tidal levels in the low-frequency range shows an increasing trend toward upstream direction. Due to the influence of the river discharge, the amplitudes of subtidal constituents in the T_TIDE model are wrongly amplified, resulting in the low accuracy of the T_TIDE model in the upper reach of the Yangtze estuary.

Compared with the T_TIDE model, the NS_TIDE model can directly represent the influence of the river discharge on estuarine tides. The regression approach used in the NS_TIDE model has finer resolution in frequencies than the signal analysis approach; however, due to the assumption of the stage model, the NS_TIDE model does not well represent the effect of the river discharge on the subtidal fluctuation of tidal levels in the Yangtze estuary. The Fourier analysis of residuals confirms that the errors of NS_TIDE mainly come from the subtidal band. By replacing the stage model with the frequency-expanded tidal-fluvial model, the MNS_TIDE model can represent the signal variance in the low-frequency band better than the NS_TIDE model; thus, the accuracy of the MNS_TIDE model is significantly increased.

As illustrated by Matte et al. (2013), the discharge terms $c_{k, 1} Q_{R}^{p_{f}}\left(t-t_{Q}\right)$ and $s_{k, 1} Q_{R}^{p_{f}}\left(t-t_{Q}\right)$ in Eqs. (7) and (8) are usually opposite in phase with terms $c_{k, 0}$ and $s_{k, 0}$, respectively, which represents a correction to $c_{k, 0}$ and 

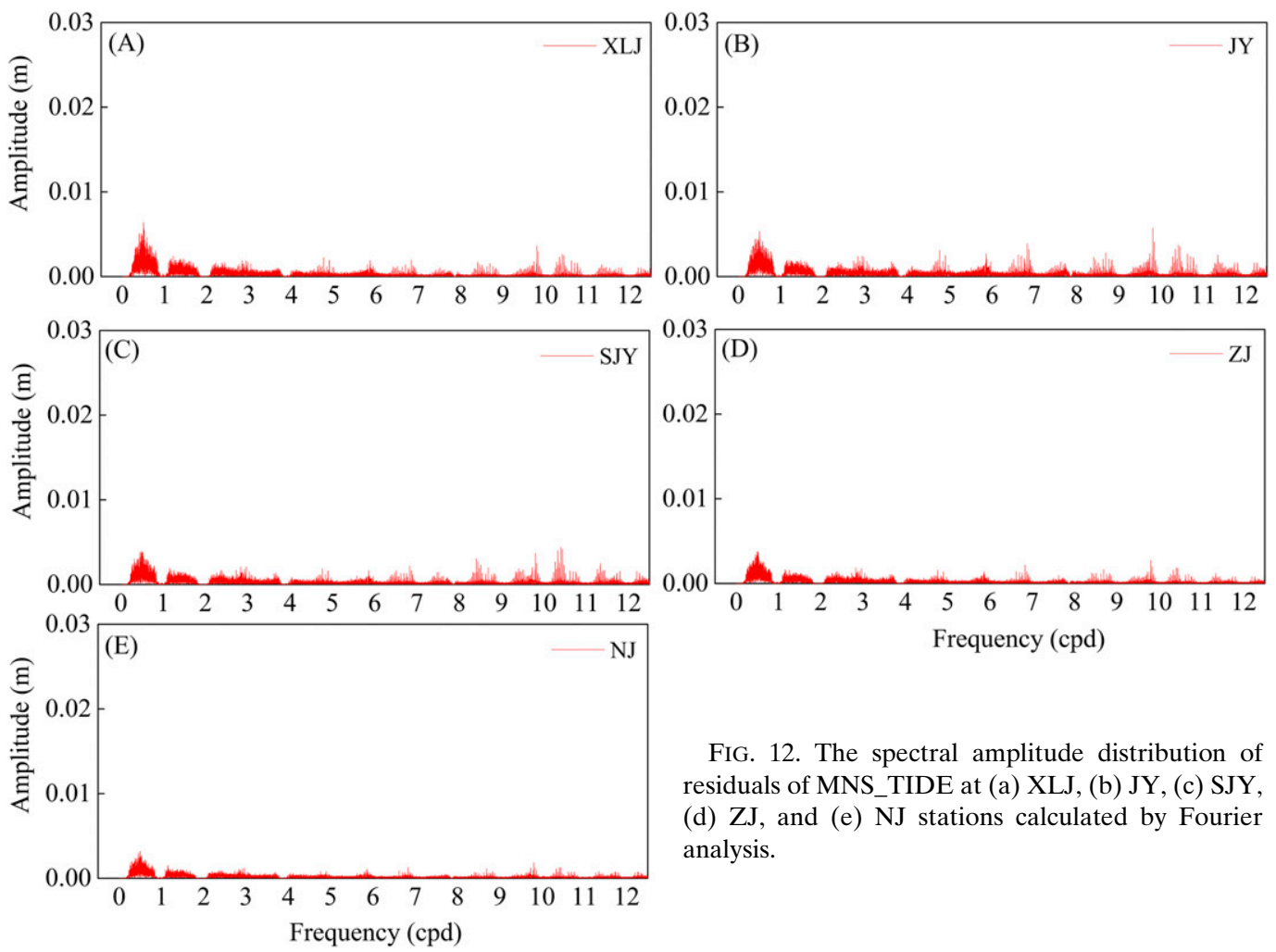

FIG. 12. The spectral amplitude distribution of residuals of MNS_TIDE at (a) XLJ, (b) JY, (c) SJY, (d) ZJ, and (e) NJ stations calculated by Fourier analysis.

$s_{k, 0}$. When the river discharge is very strong, the "artificial" tidal amplitude (Matte et al. 2013) may be generated. Therefore, the coefficients regressed in the analysis period may not be able to represent the coefficients in the forecasting period, resulting in the accuracy of forecasting tidal levels being lower than the one in the analysis period. The improved method proposed by Matte et al. (2013) was tested in the MNS_TIDE model, but it failed to reduce the appearance of the "artificial" tidal amplitude. Furthermore, as more tidal constituents are included in the MNS_TIDE model, the degree of freedom is also increased, which may cause the overfitting problem. This can also affect the performance of the MNS_TIDE model in the forecasting period. Nevertheless, the results in this study clearly show that the MNS_TIDE model can significantly improve the hindcast (herein named as prediction) accuracy of tidal levels in the Yangtze estuary.

The availability of continuous hourly tidal levels is important for estuarine studies (Supharatid 2004). Not only could it be used for the verification of hydrodynamic models in the estuaries, but also, it could provide the essential boundary conditions for the application of those models. However, due to some reasons such as instrumentation adjustment or instrument failure (Hsieh and Pratt 2001), the continuous hourly tidal levels with high quality may not be available all the time. The
MNS_TIDE model could serve as a useful tool to recover the missing tidal-level points or modify the abnormal tidal levels at the estuaries where the river discharge and the astronomical tides are both strong.

\section{Conclusions}

In this study, an improved nonstationary tidal harmonic analysis approach, namely, the MNS_TIDE model, is developed for the analysis of estuarine tides in the Yangtze estuary. In comparison with the T_TIDE and NS_TIDE models, the MNS_TIDE model can achieve a significant improvement of the nonstationary tidal harmonic analysis of estuarine tides in the Yangtze estuary.

The results clearly show an unsatisfactory performance of the T_TIDE model in analyzing the mesotides in the upper reach of the Yangtze estuary, mainly due to the incorrect representation of nonstationary tidal constituents influenced by the river discharge. Although the NS_TIDE model is more capable of tackling the nonstationary tides, it is still unable to well represent the subtidal fluctuation in the Yangtze estuary to the required accuracy. In comparison with the NS_TIDE model, the MNS_TIDE model provides an improved capability of fully representing the tidal signal variance of the Yangtze estuary, especially in 
the subtidal frequency band. The MNS_TIDE model yields a high accuracy, with the RMSE value in the range of only $0.04-0.06 \mathrm{~m}$ for the data measured at all hydrographic stations along the Yangtze estuary. Therefore, the MNS_TIDE model is well suited for the tidal analysis in the mesotidal estuaries where both the river discharge and tides are strong. The MNS_TIDE model can also provide some other promising functions such as data recovery and abnormal data point detection of the tidal levels in the estuaries.

Acknowledgments. This work was partly supported by the National Key R \& D Program of China (2017YFC0405401), the Fundamental Research Funds for the Central Universities of China (2017B20214, 2018B635X14), and the Postgraduate Research and Practice Innovation Program of Jiangsu Province (KYCX18_0602). Thanks also go to Pascal Matte for providing the NS_TIDE software package. The private communications with Pascal Matte and Haidong Pan were very helpful for the improvement of the quality of this manuscript. The authors would also like to thank the anonymous reviewers for their constructive comments.

\section{REFERENCES}

ASCE, 2000a: Artificial neural networks in hydrology. I: Preliminary concepts. J. Hydrol. Eng., 5, 115-123, https://doi.org/ 10.1061/(ASCE)1084-0699(2000)5:2(115).

— 2000b: Artificial neural networks in hydrology. II: Hydrologic applications. J. Hydrol. Eng., 5, 124-137, https://doi.org/ 10.1061/(ASCE)1084-0699(2000)5:2(124).

Cai, H., Q. Yang, Z. Zhang, X. Guo, F. Liu, and S. Ou, 2018: Impact of river-tide dynamics on the temporal-spatial distribution of residual water level in the Pearl River channel networks. Estuaries Coasts, 41, 1885-1903, https://doi.org/10.1007/ s12237-018-0399-2.

Chang, F. J., and Y. C. Chen, 2003: Estuary water-stage forecasting by using radial basis function neural network. J. Hydrol., 270, 158-166, https://doi.org/10.1016/S0022-1694(02)00289-5.

Chang, H. K., and L. C. Lin, 2006: Multi-point tidal prediction using artificial neural network with tide-generating forces. Coastal Eng., 53, 857-864, https://doi.org/10.1016/j.coastaleng.2006.05.001.

Chen, W., K. Chen, C. P. Kuang, D. Z. Zhu, L. L. He, X. D. Mao, H. D. Liang, and H. L. Song, 2016: Influence of sea level rise on saline water intrusion in the Yangtze River estuary, China. Appl. Ocean Res., 54, 12-25, https://doi.org/10.1016/ j.apor.2015.11.002

Codiga, D. L., 2011: Unified tidal analysis and prediction using the UTide MATLAB functions. University of Rhode Island Graduate School of Oceanography Tech. Rep. 2011-01, 59 pp., ftp://www.po.gso.uri.edu/pub/downloads/codiga/pubs/2011CodigaUTide-Report.pdf.

Flinchem, E. P., and D. A. Jay, 2000: An introduction to wavelet transform tidal analysis methods. Estuarine Coastal Shelf Sci., 51, 177-200, https://doi.org/10.1006/ecss.2000.0586.

Foreman, M. G. G., 1977. Manual for tidal heights analysis and prediction. Institute of Ocean Sciences Pacific Marine Science
Rep. 77-10, 97 pp., http://www.omg.unb.ca/GGE/5013_LABS/ heights.pdf.

Gallo, M. N., and S. B. Vinzon, 2005: Generation of overtides and compound tides in Amazon estuary. Ocean Dyn., 55, 441-448, https://doi.org/10.1007/s10236-005-0003-8.

Guo, L. C., V. D. W. Mick, D. A. Jay, P. Matte, Z. B. Wang, D. Roelvink, and Q. He, 2015: River-tide dynamics: Exploration of nonstationary and nonlinear tidal behavior in the Yangtze River estuary. J. Geophys. Res. Oceans, 120, 34993521, https://doi.org/10.1002/2014JC010491.

Hoitink, A. J. F., and D. A. Jay, 2016: Tidal river dynamics: Implications for deltas. Rev. Geophys., 54, 240-272, https:// doi.org/10.1002/2015RG000507.

Holland, P. W., and R. E. Welsch, 1977: Robust regression using iteratively reweighted least-squares. Commun. Stat., 6, 813827, https://doi.org/10.1080/03610927708827533.

Hsieh, B. B., and T. C. Pratt, 2001: Field data recovery in tidal system using artificial neural networks (ANNs). U.S. Army Corps of Engineers Tech. Note ERDC/CHL CHETN-IV-38, 10 pp.

Jay, D. A., 1991: Green's law revisited: Tidal long-wave propagation in channels with strong topography. J. Geophys. Res., 96, 20 585-20 598, https://doi.org/10.1029/91JC01633.

_ , and E. P. Flinchem, 1999: A comparison of methods for analysis of tidal records containing multi-scale non-tidal background energy. Cont. Shelf Res., 19, 1695-1732, https:// doi.org/10.1016/S0278-4343(99)00036-9.

— analysis-The uses of non-stationary data. Ocean Dyn., $\mathbf{5 3}$ 110-125, https://doi.org/10.1007/s10236-003-0042-y.

—, K. Leffler, and S. Degens, 2011: Long-term evolution of Columbia River tides. J. Waterw. Port Coastal Ocean Eng., 137, 182-191, https://doi.org/10.1061/(ASCE)WW.1943-5460.0000082.

Khan, M. Y. A., F. Hasan, S. Panwar, and G. J. Chakrapani, 2016: Neural network model for discharge and water level prediction for Ramganga River catchment of Ganga basin, India. Hydrol. Sci. J., 61, 2084-2095, https://doi.org/10.1080/ 02626667.2015.1083650.

Kukulka, T., and D. A. Jay, 2003a: Impacts of Columbia River discharge on salmonid habitat: 1 . A nonstationary fluvial tide model. J. Geophys. Res., 108, 3293, https://doi.org/10.1029/2002JC001382.

$\longrightarrow$, and $-2003 \mathrm{~b}$ : Impacts of Columbia River discharge on salmonid habitat: 2. Changes in shallow-water habitat. J. Geophys. Res., 108, 3293, https://doi.org/10.1029/2002JC001382.

Leffler, K. E., and D. A. Jay, 2009: Enhancing tidal harmonic analysis: Robust (hybrid L1/L2) solutions. Cont. Shelf Res. 29, 78-88, https://doi.org/10.1016/j.csr.2008.04.011.

Liang, S. X., M. C. Li, and Z. C. Sun, 2008: Prediction models for tidal level including strong meteorologic effects using a neural network. Ocean Eng., 35, 666-675, https://doi.org/ 10.1016/j.oceaneng.2007.12.006.

Lu, S., C. F. Tong, D. Lee, J. H. Zheng, J. Shen, W. Zhang, and Y. X. Yan, 2015: Propagation of tidal waves up in Yangtze estuary during the dry season. J. Geophys. Res. Oceans, 120, 6445-6473, https://doi.org/10.1002/2014JC010414.

Matte, P., D. A. Jay, and E. D. Zaron, 2013: Adaptation of classical tidal harmonic analysis to nonstationary tides, with application to river tides. J. Atmos. Oceanic Technol., 30 569-589, https://doi.org/10.1175/JTECH-D-12-00016.1.

_- Y. Secretan, and J. Morin, 2014: Temporal and spatial variability of tidal-fluvial dynamics in the St. Lawrence fluvial estuary: An application of nonstationary tidal harmonic analysis. J. Geophys. Res. Oceans, 119, 5724-5744, https:// doi.org/10.1002/2014JC009791. 
, and - 2018: Reconstruction of tidal discharges in the St. Lawrence fluvial estuary: The method of cubature revisited. J. Geophys. Res. Oceans, 123, 5500-5524, https:// doi.org/10.1029/2018JC013834.

Pan, H. D., Z. Guo, Y. Y. Wang, and X. Q. Lv, 2018a: Application of the EMD method to river tides. J. Atmos. Oceanic Technol., 35, 809-819, https://doi.org/10.1175/JTECH-D-17-0185.1.

- X. Q. Lv, Y. Y. Wang, P. Matte, H. B. Chen, and G. Z. Jin, 2018b: Exploration of tidal-fluvial interaction in the Columbia River estuary using S_TIDE. J. Geophys. Res. Oceans, 123, 6598-6619, https://doi.org/10.1029/2018JC014146.

Pawlowicz, R., B. Beardsley, and S. Lentz, 2002: Classical tidal harmonic analysis including error estimates in MATLAB using T_TIDE. Comput. Geosci., 28, 929-937, https://doi.org/ 10.1016/S0098-3004(02)00013-4.
Supharatid, S., 2004: Field data recovery of tidal level using a neuro-genetic algorithm. Coastal Eng. J., 46, 369-383, https:// doi.org/10.1142/S0578563404001117.

Tan, Y., F. Yang, and D. H. Xie, 2016: The change of tidal characteristics under the influence of human activities in the Yangtze estuary. J. Coastal Res., 75, 163-167, https://doi.org/ 10.2112/SI75-033.1.

Tsai, C. P., and T. L. Lee, 1999: Back-propagation neural network in tidal-level forecasting. J. Waterw. Port Coastal Ocean Eng., 125, 195-202, https://doi.org/10.1061/(ASCE) 0733-950X(1999)125:4(195).

Zhang, W., Y. Cao, Y. L. Zhu, J. H. Zheng, X. M. Ji, Y. W. Xu, Y. Wu, and A. J. F. Hoitink, 2018: Unravelling the causes of tidal asymmetry in deltas. J. Hydrol., 564, 588-604, https:// doi.org/10.1016/j.jhydrol.2018.07.023. 